\title{
EL «DESPERTAR» DE LA MUJER NORTEAMERICANA: CREACIÓN DE UNA ESTÉTICA FEMINISTA EN THE AWAKENING DE KATE CHOPIN
}

\author{
marfa ANTONIa Álvarez Calleja
}

UNED

En The Awakening (1989), Kate Chopin (1851-1904) va presentándonos gradualmente el despertar de su heroína Edna Pontellier: en primer lugar es un despertar doméstico y prosaico, por medio de la aparición de una sensación de descontento, al experimentar la indescriptible opresión que supone su confinamiento en un recinto donde tan sólo se espera que desempeñe el papel de madre. Edna despierta de los sueños románticos de la adolescencia para encontrarse ya una mujer casada y ver que el significado del matrimonio es muy diferente de lo que se había imaginado. Si Kate Chopin utiliza la descripción de ese entorno doméstico que hace a Edna sentirse confinada, es para que sea posible otro despertar más fantástico y poético, en el que la heroína descubre su posición en el universo; el despertar triunfante de un alma solitaria más allá de la cultura patriarcal. No obstante, como afirma Elaine Schowalter, Edna despierta a un mundo que no ofrece lugar para la mujer en que ella desea convertirse (1979: 31), y por eso Sandra M. Gilbert y Susan Gubar (1984: 408) creen que es peligroso el despertar de Edna Pontellier, la heroína de Kate Chopin, precisamente porque comprende que nunca podrá llegar a estar más cerca de alcanzar la verdadera vida que ansía: 
A certain light was beginning to dawn dimly within her - the light which, showing the way, forbids it. At that early period it served but to bewilder her. It moved her to dreams, to thoughfulness, to a shadowy anguish... was beginning to realize her position in the universe as a human being, and to recognize her relations as an individual to the world within and about her. This may seen like a ponderous weight of wisdom to descend upon the soul of a young woman. (p. 57)

The Awakening ha sido considerada por la crítica como una nueva version norteamericana de Madame Bovary, de Flaubert: Edna Pontellier, al igual que Emma Bovary, se va apartando de su marido, va desatendiendo a sus hijos, se enamora, y ante la imposibilidad de realizar sus sueños, finalmente decide abandonarlo todo.

Comprende que el lado físico del amor tiene cabida junto al espiritual, y que el sexo es una fuerza básica que -encubierta con emociones románticasconduce ciegamente hacia la procreación. Se da cuenta de que le resulta imposible volver a la sumisión del pasado. No puede consentir que sus hijos «drag her into the soul's slavery for the rest of her days» (p. 175) y, finalmente, acepta la responsabilidad de no manchar su nombre y desaparece.

Desde el principio la crítica dejó bien claro que no consideraba digna de publicarse esta obra maestra de Chopin, ya que creía innecesario que una escritora de tal gracia y finura tuviera que penetrar en el campo del sexo. Incluso en las bibliotecas de San Luis, su tierra natal, se prohibi6 el libro, a pesar de que Chopin trat 6 de defenderse por todos los medios a su alcance; incluso escribiendo esta apología:

Having a group of people at my disposal, I thought it might be entertaining to throw them together and see what would happen. I never dreamed of Mrs Pontellier making such a mess of things and working out her own damnation as she did. If I had had the slightest intimation of such a thing I would have excluded her from the company. (1969: 9)

Pero, se pregunta Helen Taylor, ¿por qué recibió esta obra universalmente una acogida tan negativa? La principal razón que ofrece la crítica contemporánea es que la heroína de Kate Chopin 
... shows a concern for self-fulfilment at the expense of her duties to husband and children, an outrageous abnegation of responsibility in a society which saw woman's primary obligation as the requirements of hearth and home. (1983: XVI)

La sociedad norteamericana de la década de 1890 podía tratar de acomodarse a las demandas de la new woman en cuanto a una mejor educación, derecho al trabajo y al voto. Lo que no podía tolerar era su deseo de libertad y satisfacción sexual, especialmente fuera del matrimonio. El sexo era una de las obligaciones de la vida matrimonial -incluso en la relativamente permisiva sociedad criolla de Nueva Orleans-y por ello se recompensaba a la mujer con el soporte económico del marido y su colocación en un pedestal, desde el que proclamaba su castidad y fidelidad. El Sur se adhería a esta idealización de la mujer de forma más rígida que el Norte, y la mujer sureña - especialmente la «mujer-madre» descrita en The Awakening - personificaba, tanto para el yanqui como para el sureño, la virtud, lealtad y fuerza de los lazos familiares que los norteamericanos esperaban reunieran sus mujeres. De ahí que la censura impuesta a The Awakening tenga como causa principal el que Edna Pontellier desafiara las convenciones sociales y sexuales: Edna rechaza su papel de mujer-madre, se entrega según su propia elección y termina afirmando que la satisfacción sexual no se corresponde necesariamente con el amor romántico. Pero, además, está segura de tener el mismo derecho que el hombre a conocerse a sí misma y a elegir su propio destino.

Sin embargo, Annette Kolodny sugiere que quizá la causa principal del rechazo de The Awakening no fuese tanto tratar el tema de la sensualidad femenina o del sexo fuera del matrimonio, como un desvío radical al elaborar sus materiales de las normas aceptadas para la novela femenina y de las que, en general, sus lectores derivaban sus expectativas. (1986: 50) Este punto es básico, ya que afecta a otras escritoras: las mismas dificultades tuvo Charlotte Perkins Gilman para ver publicada su novela corta «The Yellow Wallpaper» (1892), aunque en un contexto diferente - como apunta también Kolodny- ya que la historia no se sitúa como una desviación de la tradición anterior de la novela femenina, sino por el contrario, como una continuación del género gótico popularizado por Poe. (1986: 50) Puesto que los norteamericanos ya estaban tan acostumbrados a seguir los procesos de crisis nerviosa que presenta Edgar Alan Poe, podrían haber estado preparados para comprender a Gilman sus fantasías de reclusión y liberación, que progresivamente van debilitando la lucidez mental de la heroína: 
Might note, of course, a fleeting resemblance between the upstairs chamber in Gilman - with its bed nailed to the floor, its windows barred, and metal rings fixed to the walls - and Poe's evocation of the dungeon chambers of Toledo; in fact, a credible argument might be made for reading "The Yellow Wallpaper» as Gilman's willful and purposeful missprison of «The Pit and the Pendulum». (1986: 51).

No obstante, el lector contemporáneo de «The Yellow Wallpaper», al parecer, no estaba preparado para establecer estas conexiones, debido a que la escritura femenina tenía delimitada su visión de la sociedad a ciertos temas considerados propios de la mujer. Pero, tanto Chopin como Gilman se anticiparon a su época, negándose a escribir dentro de esas convenciones establecidas. Desde 1840 hasta 1880 - según Catherine Belsey y Jane Morre (1989: 8)— las mujeres escribían básicamente imitando los modelos masculinos, aunque los temas fueran claramente femeninos, a pesar de que la inauguración oficial del Movimiento Feminista en Norteamérica tuvo lugar en 1848. Nina Baym se expresa en el mismo sentido, asegurando que «women's fiction was an identifiable genre, one that focussed on the trials and tribulations of a noble heroine winning her own way in the world» (1978: 11), debiendo ajustarse a las normas fijadas: el ideal femenino era una mujer delicada, que sabía entretener de una manera inteligente y mostrar su modestia tanto en la forma de hablar como en los temas elegidos. Es decir, en la época en que Kate Chopin comenzó a escribir, ya existía una gran tradición de conocidas escritoras, algunas de ellas prolíficas, pero la mayoría ha pasado ya al olvido. Nombres como Fanny Fern, Elizabeth Oakes Smith o Ann Sthephens hoy sólo pueden interesar a algún historiador de la literatura, aunque sus libros inundaban el mercado cuando salían a la luz: Susan Warner publicó The Wide, Wide World en 1850, y para finales de siglo había vendido más de un millón de ejemplares; la primera novela de Marion Harland, Alone, apareció en 1854, y estuvo vendiendo 100.000 ejemplares durante varios años; Tempest and Sunshine, de Mary Jane Holmes, también de 1854, se estuvo reeditando hasta 1907; pero incluso novelas tan leídas como St. Elmo (1866), de Augusta Jane Evans, nadie las recuerda. Harriet Beecher Stowe, con La Cabaña del tío Tom (1851: 2) fue, sin embargo, la primera norteamericana que sobrepasó el ámbito nacional como «bestseller» y cuya fama perdura.

Hacia 1870, después de la Guerra Civil, el interés por la novela sentimental disminuyo, apareciendo la figura de la escritora «colorista local», especialmente de novela corta, una forma literaria que llegó a adquirir gran prestigio en esta época. Este tipo de literatura centraba su interés en una región determinada 
de los EE.UU., normalmente rural, y describía la lengua, las costumbres y la indiosincrasia de sus gentes, a menudo de forma idealizada y nostálgica, por ejemplo, Sarah Orne Jewett (1849-1909), con su descripción del Maine al comienzo de «By the Morning Boat» (1896):

On the coast of Maine, where many green islands and salt inlets fringe the deepcut shore line; where balsam first and bayberry bushes send their fragance far seaward... and the tide runs plashing in and out among the weedy ledges... On the lonely coast of Maine stood a small gray house facing the morning light. All the weather-beaten houses of that region face the sea apprehensively, like the women who live in them.

Ya desde finales de la década de 1860 empezó a formarse un grupo de escritoras cuyas novelas tenían como tema principal la liberación de la mujer. Elaine Showalter divide el movimiento feminista en tres fases - feminine, feminist and female - y, en la segunda, que abarca desde 1880 hasta 1920, afirma que

... women are historically enabled to reject the accomodating postures of femininity and to use literature to dramatise the ordeals of wronged womanhood. The personal sense of injustice which feminine novelists expressed in their novels of class struggle and factory life become incresingly and explicity feminist in the 1880 s. When a generation of $\mathrm{New}$ Women redefined the woman artist's role in terms of responsibility to suffering sisters. (1979: 35-6)

Como ocurre en «The Yellow Wallpaper» y en The Awakening, donde Gilman y Chopin tratan de descifrar la relación entre opresión y liberación; en el primer caso por medio de la locura y en el segundo, mediante el suicidio.

Por tanto, el despertar de la mujer norteamericana puede situarse claramente en las últimas décadas del siglo XIX. Particularmente desde 1880, las escritoras formulaban protestas y demandas claramente feministas, y durante la década de 1890, la new woman se había convertido en un tema central. La masacre producida por la Guerra Civil forzó a muchas mujeres, que acababan de enviudar o habían sido condenadas al celibato, a empezar a trabajar en lo que se consideraban dominios masculinos, a fin de poder sobrevivir y ayudar a la economía nacional. La mayoría de las mujeres de clase media se dedicaron a la 
enseñanza, pero en 1897 un artículo publicado en la primera revista editada por una mujer en Nueva Orleans, Daily Picayune, incluía una amplia relación de profesiones a las que anteriormente se dedicaban exclusivamente los hombres y que ahora se repartían las mujeres. No obstante, trabajar fuera de casa seguían considerándose una desgracia para la mujer de clase media, cuya esfera real era el hogar, y en este marco colocan Charlotte Perkins Gilman y Kate Chopin a sus heroínas; la primera en «the nursery at the top of the house» con las ventanas de rejas y la sumisión forzosa de la mujer que debe «take great pains to control myself" ante su marido doctor, y la segunda en un entorno totalmente familiar; el lugar veraniego cuya población es básicamente femenina:

The mother-women seemed to prevail that summer at Grand Isle. It was easy to know them, fluttering about with extended, protecting wings when any harm, real or imaginary, threatened their precious brood. They were women who idiolized their children, worshiped their husbands, and esteemed it a holy privilege to efface themselves as individuals and grow wings as ministering angels. (p. 51).

Tanto Edna Pontellier como la esposa de «The Yellow Wallpaper»-es significativo que no sepamos su nombre, ya que representa un personaje opuesto a Jennie, el ángel del hogar- están decididas a no someterse fielmente ante ningún criterio autoritario. La protagonista de «The Yellow Wallpaper» declara que no está de acuerdo con las ideas de su marido, que desea liberarla de cualquier tipo de ocupación y aislarla del exterior para así proporcionarle descanso: «Personnally, I believe that congenial work, with excitement and change, would do me good» y también "go and make a visit to Cousin Henry and Julia» mientras «laid me on the bed, and sat by me and read to me till it tired my head.» Y Edna Pontellier decide que el papel exclusivo de madre no llena sus aspiraciones: «I feel like painting», afirma desoyendo las órdenes de su marido: «It seems to me the utmost folly for a woman at the head of a household, and the mother of children, to spend in an atelier days which would be better employed contriving for the comfort of her family». Las dos protagonistas tratan de escapar de un mundo que no les ofrece la posibilidad de convertirse en el tipo de mujer-artista que desean, convencidas de que el arte es el único camino para evadirse de la prisión cuidadosamente elaborada durante siglos por la sociedad patriarcal. La heroína de Charlotte Perkins Gilman, al igual que la autora (este relato es autobiográfico, como explica en "Why I Wrote "The Yellow Wallpaper"»), tienen una 
auténtica vocación de escribir, pero en la cultura patriarcal occidental, como afirman Sandra M. Gilbert y Susan Gubar (1979: 6), el autor del texto es el padre, progenitor, procreador, patriarca estético cuya pluma es un instrumento de poder general.

Tanto Gilman como Chopin aportaron un esfuerzo importante al movimiento feminista a finales del siglo XIX y comienzos del XX. Gilman fue escritora, editora, conferenciante y organizadora eficaz, $y$ ahora podemos claramente apreciar su contribución a la liberación de la mujer: Women and Economics (1898) es un «manifiesto feminista» donde expone la tesis que defendió durante toda su vida sobre la independencia económica de la mujer, ampliada posteriormente en Concerning Children (1900), The Home (1904) y Man-Made World (1911), donde resalta la competitividad y agresividad de los hombres. Sobre Kate Chopin, a pesar de las tendencias que claramente muestra en su obra, no coinciden las opiniones de la crítica sobre los objetivos e intensidad de su feminismo: "Kate Chopin's criticism has been slow to mature from discussions of the author's unusual, sometimes revolutionary themes to analyse the craft behind these themes" (Lattin, 1980: 19). Judie Newman recuerda que Kate Chopin no tomó parte en el movimiento de emancipación de la mujer, añadiendo que «she was happily married and devoted to her six children» (1985: 152). Lo que no hay duda es que sus relatos muestran toda la compleja problemática femenina de su época - «her stories suggest that women are amply capable of enlarging the roles available to them, and that active accomodation transcends empty conformity or withdrawal into one or another pre-ordained fantasy» (Lattin, 1980: 19) - y en sus personajes femeninos hay «a clear drive toward autonomy.» (Arner, 1970: 39)

Este esquema se repite en la mayoría de sus relatos: en «Emancipation» se reimagina a sí misma como una bella bestia escapando de su confortable jaula en una alocada carrera hacia lo desconocido. Y «Elizabeth Stock's One Story" (1898) proporciona un modelo del silencio femenino que sugiere incluso mayor vulnerabilidad al ataque de la autoridad masculina. Lo más notable de su heroína es que casi parece anticiparse a su suerte reprimiendo anticipadamente sus deseos. Claro que el personaje de Chopin cuenta una historia, pero es la narración de su complicidad en el complot masculino, que trata de privarla de su profesión, su posición e incluso su vida. Elizabeth, una encargada de la oficina de correos de una pequeña ciudad, en cierto sentido disfruta de un status envidiable y poco corriente para una soltera de 38 años que, además, no siente miedo ni verguenza de serlo. Siempre había deseado ser escritora, aunque empieza su relato admitiendo que «no tiene estilo» y, peor aún, «no encuentra un argumento», a pesar de tener acceso a la sucesión de historias escritas en las postales que no puede evitar leer. $Y$ al descubrir en una de ellas una citación urgen- 
te, se expone a ponerse enferma al llevarla en mano, a pesar de la tormenta, hasta la mansión señorial de la ciudad, para entregarla al influyente destinatario. Pero el resultado es desastroso, ya que enferma gravemente y acaban quitándole su empleo para dárselo a un joven. El tema, por tanto, trata de la derrota femenina en la batalla de los sexos.

En «The Story of an Hour», la historia retrata a una esposa que muere de dolor cuando descubre que su marido no ha muerto en un accidente, lo que le impedirá volver a sentir esa libertad que ha disfrutado durante una hora. Su análisis de la forma en que las mujeres se ven a sí mismas $\longrightarrow$ permiten que los demás las vean- es sutil y lleno de implicaciones, ofreciendo el retrato más sorprendente de la aspiración femenina de libertad. Per Seyersted considera esta obra el «cuadro más sorprendente de autoafirmación femenina» (1969: 111), donde la protagonista trata de olvidar su vida pasada, cómoda y feliz, pero oscura, que jamás le había permitido llegar a realizarse plenamente. La señora Mallard, enferma del corazón, llora «with sudden, wild abandonment» por la noticia de la muerte de su esposo en un accidente, pero cuando «the storm of grief had spent itself», sola en su habitación, siente que algo nuevo y diferente se aproxima hacia ella, algo que va a cambiar su vida. Louise Mallard experimenta un momento de éxtasis:

There was something coming to her and she was waiting for it, fearfully. What was it? She did not know; it was too subtle and elusive to name. But she felt it, creeping out of the sky, reaching toward her through the sounds, the scents, the color that filled the air. (1969: 214)

Aunque sabe que volverá a llorar por la pérdida de un esposo amable, que la amaba y que le había proporcionado una vida plena de acuerdo con los cánones femeninos de la época, la voz del narrador exclama: «What could love, the unsolved mystery, count for in face of this possession of self-assertion which she suddenly recognized as the strongest impulse of her being!» Durante esa hora en que Louise se creía viuda, había podido observarse «a feverish triumph" en sus ojos «and she carried herself unwittingly like a goddes of Victory». Pero si en la primera mitad de la historia Louise Mallard «rose because he sank», al final del relato su situación es opuesta: se derrumba porque parece que su esposo literalmente surge desde la muerte. Ha descubierto que ni el amor, ni la sensación de seguridad que le proporcionaba su matrimonio, pueden compensar la falta de iniciativa o de autocontrol de su propia existencia. De ahí que tiemble de placer al verse libre, libre en cuerpo y alma!, sin nadie 
que controle sus actos: «Free! Body and soul free! There would be no one to live for her during those coming years; she would live for herself.» El destino que parecía haberla libertado ha vuelto a esclavizarla, de ahí el tema central del matrimonio como cautividad.

En The Awakening, la última obra importante que escribió Kate Chopin, crea una protagonista multidimensional que desafía las innumerables restricciones que las expectativas y prohibiciones de la sociedad imponen a los individuos, principalmente a las mujeres.

Desde la década de 1960, la crítica de The Awakening se ha concentrado en el uso extraordinario de imágenes que aparecen en la novela; la utilización sutil del color local al dibujar la atmósfera lánguida y sensual, dentro de las rígidas costumbres de la forma de vida criolla; su análisis de la situación sin horizonte de la mujer independiente que no recibe ninguna clase de ayuda de sus semejantes. Pero especialmente el campo narrativo que ofrece el tema del mar tiene importantes consecuencias estéticas: su sonido es seductor: «never ceasing, whispering, clamoring, murmuring, inviting the soul to wander for a spell in abysses of solitude, to lose itself in mazes of inward contemplation.» (p. 57)

The Awakening comienza y termina en un pueblecito costero, lo que permite a la autora que el mar enmarque toda la acción de la obra: comienza cuando la pareja central -Edna y el joven Robert - vienen a nadar, y termina cuando la protagonista se adentra en el agua del Golfo reluciendo con un millón de rayos de sol, y las olas rompen sobre la playa en pequeñas crestas de espuma, acariciando el cuerpo desnudo de Edna e invitándola hacia el paraíso que le brinda la libertad deseada. Mucho antes ya había sentido la heroína el deseo de nadar muy lejos, hacia donde ninguna mujer había nadado antes. (p. 93)

Por otro lado, la acción de aprender a nadar por parte de la protagonista es simbólica, como indican los términos elegidos para su descripción: "someone, perhaps it was Robert, thought of a bath at that mystic hour and under that mystic moon.» (p. 72) Este lenguaje marino incluso lo utiliza Edna al recordar su niñez: «a meadow that seemed as big as the ocean», donde ella «threw out her arms as if swimming when she walked, beating the tall grass as one strikes out in the water.» (p. 70)

Aunque casi toda la obra esté contada desde la perspectiva de Edna, con algunas disgresiones del narrador, sin embargo, en el capítulo 1 la escena es realista, descrita por un narrador omniscente y desde el punto de vista del impaciente hombre de negocios, Mr. Pontelllier, quien considera a su esposa algo valioso de poseer, pero a la que no es necesario escuchar: 
«You are burnt beyond recognition,» he added, looking at his wife as one locks at a valuable piece of personal property... (p. 44)

Todo este entomo familiar para la mujer del siglo XIX - la esfera femenina o el lugar asignado ritualmente a la mujer por la cultura de la época- se va diluyendo cuando el punto de vista narrativo se desplaza gradualmente hacia la heroína. No obstante, está justificada su aparición al comienzo de la obra, pues el despertar de Edna tiene lugar en un entomo peculiar, ya que se encuentra rodeada de una población básicamente femenina, debido a que durante el período estival los marinos están ocupados en sus negocios y el hotel - gobernado por la bella viuda Madame Lebrun - ha reunido a muchas figuras femeninas que personifican los distintos tipos de mujer. Por medio de ellos, la autora hace una crítica específica de la identidad de la mujer-madre en la sociedad criolla sureña de la década de 1890 . Mujeres como Adele Ratignolle cumplen perfectamente el papel asignado a su condición femenina: tiene belleza, encanto y cualidades seductoras, pero su obsesión por la vigilancia de sus deberes de madre acrecientan la claustrofobia y desprecio que instintivamente siente Edna por ese estado mental que llama «satisfacción ciega», un estado que nunca podría admitir «el delirio de la vida». Por ello Adele - junto con los dos personajes masculinos- es crucial para la transformación que experimenta Edna. Adele existe exclusivamente a causa de sus hijos, y supone que a su amiga le ocurre lo mismo. Simplemente no puede comprender la afirmación de Edna de que daría su dinero y su vida (lo que no considera esencial) por sus hijos, pero nunca se sacrificaria ella misma. Edna ha comprendido que su yo -que va explorando, tímidamente al principio y con una confianza temeraria después- es lo más precioso que posee. Sin embargo, según el criterio de la sociedad, ese yo está desequilibrado, trastornado: según su marido le dice al médico, se ha operado en su esposa un cambio que «puede verse claramente que no es ella misma».

Este yo de Edna, según Helen Taylor (1983: XIX) es el yo de los poetas románticos, de los existencialistas del siglo XX, y también el yo que las escritoras femeninas han definido como el más inclinado a la aniquilación dentro del matrimonio y la maternidad. Si Adele es aceptada por la sociedad criolla es sólo por ser la perfecta madre de sus hijos; mientras que Edna es causa de preocupación y murmuraciones por atreverse a decir que «nunca volverá a pertenecer a nadie más que a ella misma.»

A Edna le recuerdan continuamente la compleja organización social que censurará cualquier movimiento hacia su libertad personal y cualquier síntoma de abandono de sus obligaciones como miembro de esa sociedad: después de 
las disputas familiares, Mr. Pontellier la deja sola y se marcha a jugar al billar o a cenar a su club comprobando al salir que lleva dinero, lo que reafirma su autonomía.

Esos momentos que Edna y Adele pueden disfrutar solas en la playa son interrumpidos por la llegada de la tropa de niños, recuerdo viviente de su responsabilidad. Cerca del final de la novela, el confinamiento de Adele denuncia la trampa biológica que Edna se arriesga a desafiar. Su descubrimiento implica una revalorización de todas sus relaciones, y una alienación de las más cruciales, aunque más perjudicial para ella: tiene que negarse a ser «una de las posesiones de Mr. Pontellier», añadiendo que sus hijos son «antagonistas que la han vencido.» (p. 175)

Frente a la carga que proporcionan las obligaciones sociales, el deseo de Edna es muy simple, pero lleno de dificultades: «no quiero más que vivir a mi manera», exclama. $Y$ todas las obligaciones sociales y familiares que abandona la producen, no vergüenza ni culpa, sino «fuerza y expansión como individuo», y desarrollo como artista: aprende a nadar; descubre la jubilosa intensidad del amor; en lugar de rendirse pasivamente a las insinuaciones de su marido, se vuelve sexualmente activa y responsable; afirma su independencia económica alquilando su propia casa y ganando dinero mediante la venta de cuadros y las apuestas en las carreras: la confianza que adquiere en sí misma se refleja directamente en sus acciones, relaciones y en el acto final de auto-determinación.

Hacia el final de The Awakening hay una escena de la fiesta de cumpleaños que Edna Pontellier celebra en la casa familiar, antes de abandonarla para trasladarse a la cercana casita donde espera poder encontrar su propia morada espiritual y material. Ha invitado a varios amigos a la cena y se presenta elegantemente vestida con un traje de raso y encaje dorado «del color de su piel». Edna preside la mesa, suntuosamente decorada con «velas de cera en maravillosos candelabros» y llena de «fragantes rosas». Las «altas sillas que a diario están en el comedor» para esta ocasión se arrinconan, reemplazándolas por otras más cómodas y lujosas, las mejores de la casa, mientras «delante de cada invitado lanzaba sus destellos una copa de gema granate» con un cóctel especial que parecía mágico. Ocupando la cabecera de la mesa, la propia Edna también parecía mágica: había «algo en su actitud y en toda su apariencia que sugería a la mujer regia, la que gobierna, la que supervisa, la que permanece sola.» (p. 145)

Normalmente la crítica ha interpretado esta escena como otra más entre las que forman la novela, en la que Chopin trata de describir la transfiguración romántica o la liberación social de su heroína. Sin embargo, Gilbert da una interpretación diferente, por considerar que en una novela escrita con tanta economía de medios como The Awakening, este capítulo es uno de los más 
elaborados, utilizándose incluso un lenguaje lleno de imágenes y alusiones simbólicas: en medio de la celebración nos dice el narrador que Edna siente «que la invade un tedio ancestral... un hálito helado que parece proceder del alguna profunda caverna donde resuena el gemido de la discordia». (p. 145) Todo el vocabulario trata de reproducir la sensación de misterio y lejanía en la heroína, el «agudo deseo de su espíritu que constantemente anhela la presencia del amado». Edna Pontellier se convierte en ese momento en la poderosa diosa del amor y del arte. Por eso Victor, el joven de la guirnalda exclama que «Venus surgiendo de la espuma no podría haber ofrecido un espectáculo más cautivador que Mrs. Pontellier, refulgente de belleza y diamantes, a la cabecera de la mesa.» (p. 146)

Si se compara la figura presentada por Chopin con los retratos femeninos del siglo XIX de la mujer artista, a pesar de las ambivalencias se observa un gran avance psicológico de la imaginación femenina (por ejemplo en George Elliot, la mujer artista estaba predestinada a la renunciación). Mademoiselle Resisz - la pianista que va a influir decisivamente en el despertar del deseo de la protagonista - dice a Edna que para ser artista hace falta «alma valerosa... un alma que se atreva a desafiar y resistir». Esto puede aplicarse a la misma Kate Chaplin, así como a las generaciones de mujeres artistas que la precedieron: que las mujeres modernistas y de finales de siglo pudieran incluso comenzar a imaginar éxitos e influencias en las precursoras sugiere que la tradición femenina del XIX puede haber autorizado a las escritoras del XX a cambiar la forma y el alcance de su papel en la ficción.

Debido al impacto negativo que produjo esta obra, a Kate Chopin a menudo se la ha separado de la rica cantera espiritual de la época que la creó y en la que se desarrolló, a pesar de que -como sugiere Sandra M. Gilbert (1987: 18)- Kate Chopin, al igual que Oscar Wilde, había empezado a especular sobre la naturaleza de la cultura, especialmente la naturaleza de los impulsos que podían transcender los imperativos de la moralidad del siglo XIX. Soñaba con un tercer Edén gobernado por una Venus regia - de ahí la escena de la celebración del cumpleaños de Edna, que acabamos de comentar-; un jardín sacramental, en vez de sacrílego, de las delicias terrenas; una cultura más allá de la cultura, cuya energía iba a consolidarse a partir de la liberación y la aceptación del deseo femenino. Su visión de tal diosa probablemente superaba en fuerza a la teología de sus contemporáneas feministas, que estaban revisando las ideas religiosas tradicionales para instaurar unas creencias centradas en la mujer. Además, su sentido de la sexualidad sacramental de la diosa se había fortalecido por la revisión erótica de las partidarias del amor libre femenino y por la política sexual radical de hombres como Walt Whitman. 
Kate Chopin desafiaba de forma consciente el tratamiento que se otorgaba al tema del sexo en la década de 1890 , tratando de descubrir la sensualidad y el descontento de las mujeres que las convenciones de la época rehusaban reconocer. En Female Desire, Rosalind Coward pone al descubierto la laguna que existía entre los beneficios que, según la sociedad patriarcal, disfrutaba la mujer y los que tenía realmente, añadiendo que «dissatisfaction is constantly recast as desire, as desire for something more... for the ideal» (1984: 13); pero este ideal no es siempre el mismo, sino que en muchos casos es contradictorio, ya que depende de la experiencia femenina. En su lectura crítica de las escritoras del siglo XIX, Mary Jacobus (1986: 5) emplea la teoría del subconsciente de Freud, a fin de descubrir una serie de omisiones textuales, contradicciones y dudas. Y Linda Dowling (1979) ha sugerido que estos tipos intelectuales de finales del XIX y principios del XX comparten el deseo fundamental de fin de siécle avant garde: el sueño de vivir más allá de la cultura: esto es, vivir más allá de la cultura patriarcal victoriana.

La crítica feminista se diferencia de otras escuelas contemporáneas de teoría crítica en que éstas derivan sus principios literarios de un único sistema de pensamiento que les proporciona sus ideas fundamentales. Tanto los estructuralistas con los descubrimientos lingüísticos de Saussure; la crítica psicoanalítica con Freud o Lacan; los marxistas con su doctrina, y los deconstruccionistas con Derrida han aportado importantes instrumentos analíticos para la teoría de la crítica feminista. Por ello, los intereses feministas pueden dar una nueva energía y vitalidad a los estudios literarios, siendo quizás el mayor éxito de la crítica feminista las reediciones de tantas obras ignoradas de escritoras que exigen una reformulación de los conceptos de la historia literaria norteamericana para poder comprender el desarrollo de las aportaciones de la mujer. El redescubrimiento de la obra de Kate Chopin ha sido lento e irregular. Durante cincuenta años no volvieron a publicarse ninguna de sus novelas o relatos, siendo un fenómeno reciente el entusiasmo que ha producido The Awakening. Hasta 1940 ningún crítico prestó atención a esta pequeña obra maestra hasta que Cyrille Arnavon escribió un análisis en francés, comparando su afinidad con Madame Bovary y sus logros como novela realista. Diez años más tarde, Kenneth Eble escribió en Western Humanities Review un artículo titulado "A Forgotten Novel: Kate Chopin's The Awakening». En 1962 Edmund Wilson dedicó seis páginas de su libro Patriotic Gore a esta obra, animando a Per Seyersted, el profesor noruego que estaba tratando de reunir las obras completas de Chopin y escribiendo una nueva biografía -en la anterior, publicada en 1932 por Daniel Rankin, se resaltaba principalmente el «color local» de los relatos, y se criticaba duramente The 
Awakening - siendo publicadas ambas obras en 1969 por la Universidad de Louisiana.

Desde esa época, los lectores norteamericanos se han ido interesando cada vez más por Kate Chopin, hasta el punto que en 1975 se empezó a publicar The Kate Chopin Newsletter, cada vez con mayor tirada, donde las escritoras feministas han ido descubriendo todas sus facetas y han ayudado a reestablecer a Chopin como una figura principal en la gran tradición de las escritoras norteamericanas, desde Harriet Beecher Stowe, pasando por Charlotte Perkins Gilman, Edith Wharton y Willa Carter, hasta Sylvia Plath, con cuya novela The Bell Jar ha sido comparada The Awakening.

Kate Chopin no sólo ha ocupado un lugar preferente entre las escritoras, sino que debido a la crítica entusiasta que ha merecido The Awakening y el creciente interés por el resto de su obra, se ha colocado dentro del contexto del realismo y del naturalismo norteamericano e incluso europeo. Recibió una profunda influencia de los realistas europeos Balzac, Zola, Ibsen, Tolstoy, y sus novelas cortas tienen mucha afinidad con las de Maupassant, al que admiraba profundamente y con quien se la compara actualmente. También se le ha comparado con dos grandes escritores de la época, Theodore Dreiser y Henry James. Como el naturalista Dreiser, Kate Chopin pintaba las verdades antirománticas de los hombres y las mujeres, y los factores sociales, económicos y biológicos que tallaban sus vidas, y como el realista James, se concentraba en las relaciones humanas más que en el panorama social y recordaba algo de su aguda penetración psicológica. Al igual que James, era capaz de evocar a toda la sociedad a través de la disección de una relación sexual, a pesar de la ruptura que supuso The Awakening y su novela corta «The Storm" con la mayoría de los realistas del siglo XIX, por alabar la sexualidad humana como un bien positivo.

En nuestros días The Awakening sigue teniendo un mensaje claro y directo para la crítica feminista -en su aspecto más importante de la ginocrítica o de la mujer «as literature by women» (Showalter, 1979: 25)—, ya que expresa la urgente necesidad de la mujer de luchar por el derecho a conocerse a sí misma y a encontrar su propio camino, sin importarle lo penoso que pueda resultar el proceso. The Awakening no es simplemente una novela acerca de una mujer que debería «haber filtreado menos y cuidado más a sus hijos», como escribió un crítico anónimo en 1899. El desarrollo psicologico que experimenta Edna Pontellier no puede considerarse tan sólo como un descubrimiento del amor y del sexo, ni de la posición económica real de la mujer casada en la sociedad, a pesar de que trate estos problemas, sino de una crítica específica de la identidad de la mujer-madre dentro de la sociedad criolla de la década de 1890 , un grupo con una identidad especial, que no hay que confundir con los criollos de 
la América Latina. La sociedad criolla norteamericana, dentro de la cual nació y posteriormente se casó Kate Chopin, eran los descendientes blancos de los primeros pobladores franceses y españoles de Nueva Orleans. Los auténticos criollos - ricos, aristocráticos y conservadores- habitaban en el antiguo distrito francés de Nueva Orleans, dando un aire mediterráneo a la ciudad, con su estilo de vida colorista y relajado.

Según la crítica feminista, la conclusión de la obra puede interpretarse de forma positiva, ya que a pesar de ser derrotada por su entorno social, Edna logra salir victoriosa, pues consigue su libertad interior sin traicionar las obligaciones contraídas: prefiere quitarse la vida antes de seguir comportándose de forma indigna con los que la rodean. Pero, no puede hablarse rotundamente del suicidio de la heroína a través de ese nadar sin fin, desnuda, por las aguas del Golfo. En ningún momento se nos dice expresamente que termine con la muerte, sino que también puede interpretarse -según Sandra M. Gilbert- como una resurrección, un viaje hacia un mundo imaginario, lejos de la cultura restrictiva del siglo XIX. Si tratamos de comprender los mitos que enriquecen la narrativa de Chopin, Edna podrá encontrar ese mundo en que las mujeres puedan ser tan libres como lo era la mítica Afrodita:

... because of the way it is presented to us, Edna's suicide expresses not a refusal to accomodate to reality but a subversive questioning of both reality and realism. For, swimming away from the white beach of Grande Isle, from the empty summer colony and the equally empty fictions of marriage and maternity, Edna swims, as the novel's last sentences tell us, not into death but back into the imaginative openness of her childhood. (1987: 31)

Incluso en la última frase de la novela, Edna sigue nadando, nadando hacia el infinito, mientras «there was the hum of bees, and the musky odor of pinks filled the air». Este ambiguo despertar triunfante de la heroína es uno de los motivos por los que en la última década The Awakening se ha convertido en una de las novelas norteamericanas más insistentemente analizadas, ya que supone una clara crítica de la identidad de la mujer-madre, una defensa del sexo que critica los mitos del amor victoriano, una fantasía e idealización del amorlibre de la mujer nueva de finales del siglo XIX.

En realidad, Kate Chopin puede considerarse como una mujer de la década de 1890, una escritora de fines de siglo, lo que supone asociarla con revolucionarios artísticos e intelectuales de la talla de Oscar Wilde y sus precursores 
Pater o Whitman. Para la mujer, la década de 1890 personifica la idea del «amor libre» y de la «mujer nueva», una mujer que elige ser, tanto política como profesional y emocionalmente, independiente y que sueña con una sociedad transfigurada que desprecie la opresión derivada de la distinción sexual patriarcal.

La tercera colección de novelas cortas de Kate Chopin, que fue rechazada por su editor, llevaba el título $A$ Vocation and a Voice, un adecuado epitafio. Al igual que Edna, cuando Kate Chopin finalmente encontró su verdadera vocación y su voz más apropiada, casi nadie la oyó ni la comprendió, pero al final su propio país ha reconocido que The Awakening es una novela feminista clásica y una obra de arte de la literatura norteamericana. El silencio a que Chopin se vio forzada por la persecución de que fue objeto supuso una pérdida para las letras norteamericanas del mismo orden que la de Crane y Norris, aunque con sus cuatro novelas: At Fault (1890), A Night in Acadie (1897), Young Dr. Gosse (que Chopin destruyo, probablemente en 1896), The Awakening (1898) y sus tres colecciones de novelas cortas —unas cien en total-, Kate Chopin tiene asegurado un lugar permanente en la literatura norteamericana.

En resumen, The Awakening, de Kate Chopin, es un claro ejemplo de escritura feminista, ya que toda la obra es una justificación de los derechos de la mujer y uno de los temas que analiza es la pasión prohibida, básico para la mujer de finales del siglo XIX. Pero lo que convierte The Awakening en una obra clave es que Kate Chopin crea aquí una estética feminista mientras trata de buscar, entre los mitos heredados de la historia patriarcal, la forma de presentar el erotismo de la nueva cultura. Al volver a examinar esos mitos va creando, casi sin darse cuenta y de manera inconsciente, una estructura narrativa con la que poder dramatizar de forma coherente la lucha de la mujer por conseguir su identidad, tema central de la obra. Como afirma Helen Taylor en la Introducción que escribió para la edición de The Awakening en 1978:

Lyrical, romantic, witty, angry, feminist, revolutionary: The Awakening speaks in many voices about many modes of thinking and being. Since the novel describes the growth of a woman artist, on one level it speaks of the author's own creative development and the conflicts this raised in her life. (1978: 5)

Chopin tenía mucho más que decir, pero perdió todo su valor y casi no volvió a escribir hasta su muerte en 1904. Durante casi tres décadas la novela 
ha sido ignorada, pasando ahora a ser uno de los primeros signos de liberación de la tradición y la autoridad patriarcal — de la liberación de la cárcel confortable del matrimonio burgués-, siendo su heroína Edna Pontellier una de las principales representantes del despertar de la mujer norteamericana de finales del siglo XIX. 


\section{REFERENCIAS BIBLIOGRÁFICAS}

Allen, Priscilla (1977), «Old Critics and New: The Treatment of Chopin's The Awakening». The Authority of Experience: Essays in Feminist Criticism (ed. Arlyn Diamond \& Lee R. Edwards). Amherst: University of Massachusetts Press, pp. 224-38.

ARMS, GeORge (1967), «Kate Chopin's The Awakening in the Perspective of Her Literary Career». Essays on American Literature in Honor of Jay B. Hubbell (ed. Clarence Gohdes). Durham: Duke University Press, pp. 215-28.

ARNER, RoBerT D. (1970), Music from a Father Room: A Study of the Fiction of Kate Chopin. PhD. dissertation. Pennsylvania State Univ.

BAYM, NINA (1978), Women's Fiction: a Guide to Novels by and about Women in America. 1820-1870. Ithaca, New York: Cornell

- 1984 (1981), «Melodrams of Beset Manhood: How Theories of American Fiction Exclude Women Authors». American Quarterly, 33.

Belsey, Catherine \& MOORE, JANE (1989), The Feminist Reader: Essays in Gender and the Politics of Literary Criticism. London: MacMillan Education Ltd.

CANTWELl, RoBert (1956), «The Awakening by Kate Chopin». Georgia Review, 10, Pp. $489-94$.

CHOPIN, KATE (1898), The Awakening and Selected Stories (Introduction: «The Second Coming of Aphrodite», Sandra M. Gilbert). New York: Viking Penguin.

COWARD, ROSALIND (1984), Female Desire: Women's Sexuality Today. London: Granada Publishing. 
Culley, Margaret (1976), «Edna Pontellier: A solitary Soul». The Awakening: A Norton Critical Edition. (ed. M. Culley). New York: W. W. Nortorn \& Co., pp. 224-28.

Diaz-DioCARETZ, MYRIAM (1985). Women Feminist ldentity and Society in the 1980's. Amsterdam/Philadelphia: John Benjamins Publishing Company.

Dowling, LiNDA (1979), «The Decadent and the New Woman in the 1890s». Nineteenth-Century Fiction, 33.

Eaton, Clement (1974), «Breaking a Path for the Liberation of Women in the South». Georgia Review, 28. Summer, pp. 187-99.

Eble, KenNETH (1956), «A Forgotten Novel: Kate Chopin's The Awakening». Western Humanities Review, 10, pp. 261-69.

FleTCHER, MARIE (1966), «The Southern Woman in the Fiction of Kate Chopin». Louisiana History, 7, pp. 117-32.

Gilbert, Sandra M. \& Gubar, Susan 1984 (1979), The Madwoman in the Attic: The Woman Writer and the Nineteenth Century Literary Imagination. Yale University Press.

- (1987), No Man's Land. The War of the Words, New Haven \& London: Yale University Press.

- (1989), No Man's Land. The Place of the Woman Writer in the Twentieth Century. New Haven \& London: Yale University Press.

Gilbert, Sandra M. (1983), «The Second Coming of Aphrodite: Kate Chopin's Fantasy of Desire». Kenyon Review, 5, pp. 42-56.

- 1987 (1984), Introducción a The Awakening and Selected Stories, de Kate Chopin. New York: Viking Penguin Inc.

Gilman, Charlotte Perkins 1985 (1892), "The Yellow Wallpaper». The Norton Anthology of American Literature. Volume 2. New York \& London: W. W. Norton \& Company.

- (1986), «What Do Feminist Critics Want? A Postcard from the Volcano». The New Feminist Criticism: Essays on Women Literature and Theory. London: Virago Press Limited. 
GOMEZ ReUS, TERESA (1990), «Vengando al ángel del hogar: la esposa loca como figura arquetípica en "The Yellow Wallpaper", de Charlotte Perkins Gilman». Estudios de Filología Inglesa: Homenaje al Doctor Pedro Jesús Marcos Pérez. Universidad de Alicante.

GubAR, SUSAN (1979), «Mother, Maiden, and the Marriage of Death: Women Writers and an Ancient Myth». Women's Studies, 6, pp. 301-15.

HutcheON, LindA 1991 (1989), The Politics of Postmodernism. London \& New York: Routledge.

JACOBUS, MARY (1979), Women Writing and Writing about Women. London: Croom Helm.

- (1982), «Is There a Woman in the Text?». New Literary History, 14. Fall., pp. 117-41.

- (1986), Reading Woman: Essays in Feminist Criticism. London.

JEWETT, SARAH ORNE 1988 (1886), A White Heron and Other Stories (The Collected Works of S. Jewett), US: Houghton.

KOLODNY, ANNETTE (1986), «A Map for Rereading: Gender and the Interpretation of Literary Text». The new Feminist Criticism: Essays on Women, Literature and Theory. London: Virago Press Limited.

LatTin, Patricia HoPkins (1980). «Kate Chopin's Repeating Characters». Mississippi Quarterly, 33, 19.

LEE, A. ROBERT (ed.) (1985). The Nineteenth Century American Short Story. London: Vision Press Ltd.

Miles, RoSALIND (1987), The Female Form. London \& New York: Routledge.

Newman, Judie 1985. «Kate Chopin: Short Fiction and the Arts of Subversion», The Nineteenth Century American Short Story (ed. A. Robert Lee), London: Vision Press Ltd.

Newton, JUdTH \& Rosenfelt, Deborah (eds.) (1985), Feminist Criticism and Social Change. Sex, Class and Race in Literary Culture. New York \& London: Methuen.

Rankin, Daniel S. (1932), Kate Chopin and Her Creole Stories. Philadelphia: University of Pennsylvania Press. 
SCOTT, ANNE FIROR (1962), «The New Woman in the New South». South Atlantic Quartely, 61, pp. 473-83.

Seyersted, Ped 1969. Kate Chopin: A Critical Biography. Baton Rouge: Louisiana State University Press.

Showalter, Elaine (1979), «Towards a Feminist Poetics». Women Writing and Writing about Women. Mary Jacobus (ed.). London: Croom Helms.

- (1986), «The Feminist Critical Revolution». The New Feminist Criticism: Essays on Women, Literature and Theory. London: Virago Press Limited, pp. 3-17.

- (1986), «Feminist Criticism in the Wilderness». The New Feminist Criticism: Essays on Women, Literature and Theory. London: Virago Press Limited, pp. 243-270.

Skaggs, Peggy (1975), The Man-Instinct of Possession: A Persistent Theme in Kate Chopin's Storiesm. Louisiana Studies, 14, pp. 277-85.

- (1985), Kate Chopin. Boston: Twayne Publishers.

Spangler, George (1970), «Kate Chopin's The Awakening: A Partial Dissent», Novel, 3, pp. 249-55.

SPINGer, MARLENE (1976), Edith Wharton and Kate Chopin: A Reference Guide. Boston: G. K. Hall.

TAYLOR, Helen 1983 (1978), Introducción a The Awakening de Kate Chopin. London: The Women's Press Limited.

Thorton, LAWrence (1980), «The Awakening: A Political Romance». American Literature, 52, 1, March, pp. 50-56.

TOMPXINS, JANE P. "The Awakening: An Evaluation». Feminist Studies, 3, pp. 22-29.

Tотн, EMLY (1975), "The Independent Woman and Free Love». Massachusetts Review, 16, pp. 647-64.

- (1976), «Kate Chopin's The Awakening as Feminist Criticism». Louisiana Studies, 15, pp. 241-51. 
WheELER, OTIS B. (1975), «The Five Awakenings of Edna Pontellier». The Southern Review, 11, pp. 118-28.

Wilson, EdMUnd (1962), Patriotic Gore. New York: Oxford University Press.

WOlFF, CYNTHIA Griffin (1973), «Thanatos and Eros: Kate Chopin's The Awakening». American Quarterly, 25, pp. 449-72.

Wolkenfeld, Suzanne (1976), «Edna's Suicide: The Problem of the One and the Many». The Awakening: A Norton Critical Edition. (ed. Marfaret Culler). New York: W. W. Norton \& Co., pp. 218-24.

ZIFF, LARZER (1966), The American 1890s: The Life and Times of a Lost Generation. New York: The Viking Press. 